\title{
Editorial: Prof. Wokyung Sung and pathways in biological physics
}

\author{
Rudi Podgornik
}

Received: 20 May 2014 / Accepted: 25 May 2014 / Published online: 16 July 2014

(C) Springer Science+Business Media Dordrecht 2014

Prof. Wokyung Sung, a member of the Journal of Biological Physics editorial board and former Editor-in-Chief in the period 2007-2009, is about to retire this year as a Professor of Physics at the Department of Physics of the Pohang University of Science \& Technology. It is for this special occasion and to recognize Prof. Sung's contributions to statistical biological physics, that his colleagues and friends are organizing an international conference entitled SURMOUNTING “THE INSURMOUNTABLE"-PATHWAYS of BIOLOGICAL PHYSICS to be held this summer in Pohang, South Korea, on the occasion of which the Journal of Biological Physics would like to thank Prof. Sung as a long-time member of the editorial board and a former Editor-in-Chief.

After graduating from Seoul National University, Prof. Sung finished his $\mathrm{PhD}$ studies at the State University of New York at Stony Brook, USA. He started his scientific career in the early 1980s working on transport phenomena and other statistical aspects of the physics of fluids. In the mid-1990s, he broadened his interests to other fundamental aspects of soft matter and polymer physics, but more importantly for this occasion, also to general physical approaches to biological phenomena and specifically to several fundamental problems in biological physics. He was the first to devise a statistical model for polymer translocation through a membrane pore [1]. His seminal work on this problem later engendered a whole new separate field in biological physics. The other two research areas where Prof. Sung was very active were semiflexible polymer theory, particularly in the case of confinement and as it relates to the description of DNA, and biomembrane fluctuations, electrostatic interactions, and related phenomena. He also contributed essentially to stochastic models of conductance in ion channels and to the various aspects of the theory of stochastic resonance in the context of polymer and ion-channel physics. His current research interests include the physical understanding of basic biological conformations and processes that emerge at the mesoscopic (cellular) level, the methodological questions in the statistical physics of soft matter (polymers, membranes) and stochastic phenomena (barrier crossing, stochastic resonance, and other issues of noise-assisted cooperative dynamics). His most recent paper deals with an improved mesoscale model of DNA [2], and was highlighted as "DNA helicity and elasticity explained on the nanoscale" in 11 media, such as Phys.org, Physics News, Science Daily, Science Newsline, Nanotechnology Now, etc.

R. Podgornik $(\square)$

Jožef Stefan Institute, Ljubljana, Slovenia

e-mail: rudolf.podgornik@ijs.si 
For his seminal contributions to science and in particular to biological physics, Prof. Sung was awarded a Medal of Science and Technology bestowed by the Korean Government in 2010. He also served as a Special Advisor to the Asia Pacific Center for Theoretical Physics (APCTP) in Pohang, and as a Chairman of the Statistical Physics Committee of the Korean Physical Society. Professor Sung was a Visiting Professor at Oxford University (1991), the Max-Planck Institut für Festkörperforschung, Stuttgart, Germany (1991), the National Institutes of Health, Bethesda, MD, USA (1997), the Juelich Research Center, Juelich, Germany (1999), the University of Pennsylvania, PA, USA (2000), and Brown University, Providence, RI, USA (2007-2008). One could sum up his scientific career with the motto: he is among those who bring physics to life, and life to physics.

\section{References}

1. Sung, W., Park, P.J.: Polymer translocation through a pore in a membrane. Phys. Rev. Lett. 77, 783 (1996)

2. Jeon, J., Sung, W.: An effective mesoscopic model of double-stranded DNA. J. Biol. Phys. 40, 1 (2014) 